\title{
Textile SIW Antennas as Hybrid Energy Harvesting and Power Management Platforms
}

\author{
Sam Lemey, Sam Agneessens and Hendrik Rogier \\ Department of Information Technology \\ Ghent University \\ Ghent, Belgium \\ Sam.Lemey@intec.ugent.be
}

\begin{abstract}
A compact, highly-integrated and unobtrusive wearable textile antenna system, able to establish a reliable and energy-efficient wireless body-centric communication link in the [2.4-2.4835]-GHz Industrial, Scientific and Medical band and enabling energy harvesting from three different energy sources, is presented. Our design approach relies on further extending the functionality of a carefully selected textile antenna by exploiting its surface as an energy scavenging and power management platform. More specific, two different ultra-flexible solar cells, a micro-energy cell and a flexible power management system are integrated onto a wearable substrate integrated waveguide cavitybacked textile slot antenna to enable energy harvesting from both solar and artificial light. Furthermore, thermal body energy harvesting, via an externally connected thermoelectric generator, is enabled by including an ultra-low voltage step-up converter. Measurements in four well-chosen indoor scenarios demonstrate that a hybrid energy-harvesting approach is necessary to obtain a more continuous flow and a higher amount of scavenged energy, leading to a higher system autonomy and/or reduced battery size.
\end{abstract}

Index Terms-Energy harvesting; energy management; textile antenna; wearable antenna.

\section{INTRODUCTION}

In the last years, smart fabric interactive textile (SFIT) systems are starting to find their way into a growing amount of real-life applications. These applications rely on wearable electronics to increase the functionality of the garments without adding heavy or cumbersome equipment. For instance, in critical professional applications, SFIT systems could enable monitoring, localization and coordination of first responders during rescue missions and interventions, to safe-guard their lifes and hence also the lifes of the persons in distress. Employing SFIT systems in healthcare applications could facilitate wireless monitoring of vital signs of patients in hospitals or in home-care scenarios. In addition, this technology could allow elderly people to stay longer at home which would partly resolve the future dilemma for health care services, originating from rising life expectancy of the world's population.

However, a major issue of current-generation SFIT systems concerns the limited system autonomy [1]. Lots of research efforts were already devoted to increase efficiency of the embedded electronic circuitry in order to increase system autonomy without using large and obtrusive batteries. As [2] indicates that the wireless communication unit is the major power consumer, a large increase in system autonomy could be obtained by carefully designing the textile antenna for large radiation efficiency and high gain. Moreover, multiple of such a highly-efficient textile antennas could be deployed onto the user's body to further increase autonomy [3]. In addition, many physiological signals that are monitored by sensors in SFIT systems change slowly. Hence, they allow burst measurement and transmission, resulting in low duty cycle operation, yielding a low average power consumption and making SFIT systems prime candidates to be partly, or even solely, powered by energy harvesting techniques [4]. For instance, energy could be extracted from ambient sources at the place where the SFIT is worn by the user [5], [6], or from the user's activities [7], [8]. Preferably, energy is scavenged from multiple diverse energy sources [4], [5] to be able to harvest more energy in a more continuous way. Anyway, a power management system is necessary to convert the small and highly fluctuating levels of harvested energy into a form useful for powering the SFIT. Although garments provide sufficient surface to integrate a textile (multi-)antenna system, a considered strategy is requisite for the integration of multiple energy harvesters, and the corresponding power management module in order to scavenge sufficient energy, on the one hand, and to maintain the user's comfort, on the other hand, without affecting antenna performance.

Therefore, in this paper, we investigate the potential of exploiting the textile antenna as an integration platform for energy harvesting hardware. In a first step, we select an antenna topology that exhibits a very high isolation from its environment, enables unobtrusive integration and facilitates integration of additional hardware. Then, we discuss the integration of a flexible hybrid energy harvesting system onto the selected textile antenna, enabling energy scavenging from three different energy sources. More specific, a flexible central power management system (CPMS), including a microenergy cell (MEC), is placed on the antenna's backside. The CPMS allows energy harvesting from indoor artificial light and outdoor solar light by integrating two different flexible solar cells on top of the textile antenna. Furthermore, thermal body energy harvesting is enabled by including a thermoelectric generator (TEG), placed with its hot side on the human skin, and extending the CPMS with an ultra-low voltage step-up convertor. Measurements performed in [4] have been extended with additional measurements in four well-considered realistic indoor environments in order to emphasize the advantages and the importance of hybrid energy harvesting. 


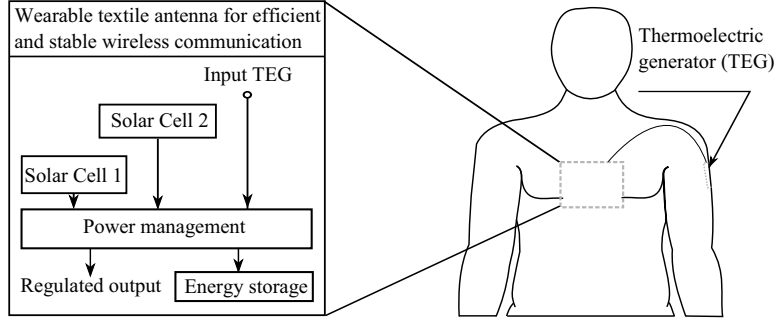

Fig. 1. Block schematic representation of system concept and application.

\section{Antenna/Harvester Co-Design}

We design a compact, highly-integrated and unobtrusive unit that is able to set-up a robust, stable and energy-efficient wireless communication link, on the one hand, and able to harvest energy from three different energy sources, on the other hand, as schematically outlined in Fig. 1. It relies on reusing the textile antenna's surface as an energy harvesting and power management platform that enables combining the energy scavenged by two different incorporated flexible solar cells and an externally connected TEG in order to provide a regulated output for adequately powering the SFIT. By simultaneous extracting energy from diverse energy sources, a higher total harvested energy can be obtained and the time, during which no energy is scavenged, can be reduced.

\section{A. Textile antenna as integration platform}

As a starting point, an antenna topology is selected that facilitates the integration of additional electronic hardware without degrading its own performance, in the meantime guaranteeing the wearer's comfort. In [4], the potential of multiple antenna topologies as integration platform is discussed. As [4] identifies SIW cavity-backed slot antennas as ideal candidates, the wearable SIW cavity-backed textile slot antenna, presented in [9], is selected to form the base of our compact, unobtrusive and wearable hybrid energy harvesting textile antenna system. However, depending on the application, other wearable textile SIW cavity-backed slot antennas, as the ones discussed in [10] may also serve as an integration platform. The selected antenna is shown in Fig. 2 and consists of a square SIW cavity in which a dog-bone shaped slot is cut out in the top metal layer. The dimensions of the cavity were selected to excite the TE120 cavity mode at $2.45 \mathrm{GHz}$, whereas the dimensions of the slot and its position were chosen to obtain optimal coupling with the excited mode. The length of the grounded coplanar waveguide, used as a feed line, was optimised to improve input matching [9] within the $2.45 \mathrm{GHz}$ ISM-band. The antenna's cavity-backed slot topology, leveraging a ground plane, in combination with the SIW technology, exploiting copper eyelets to implement the conductive walls of the backed cavity, increase the isolation between the radiating element and its environment. This makes the selected antenna not only very suitable for on-body deployment, but also extremely suitable for the integration of additional electronic circuitry. Furthermore, the materials from which the on-body antenna was assembled, were selected with greatest care to achieve a flexible, low-profile and conformal design. A closed cell expanded rubber protective foam, typically used in fire fighter jackets, is adopted as antenna substrate, whereas the antenna's slot and feed layer are constructed out of a copper-coated nylon fabric. Both conductive fabric layers are glued to the antenna substrate with thermally-activated adhesive sheets. Section II-B expands on the third reason to adopt a SIW cavity-backed slot textile antenna, being the ease by which additional hardware can be integrated and the ability to minimize the amount and length of interconnections by exploiting the properties of the topology. More details about the selected antenna can be found in [9].

\section{B. Energy harvesting and power management hardware}

The textile antenna is then exploited as an hybrid energyharvesting and power management platform, by gluing a flexible power management module and a $170 \mu \mathrm{m}$-thick MEC at its feed plane and two ultra-thin, flexible a-Si:H solar cells at its slot plane by means of stretchable adhesive sheets. The proposed power management system is built up from two interconnected subsystems, being the low power system (LPS) and the CPMS. The CPMS is based on the MAX17710 IC from Maxim Integrated and is designed to charge the MEC from two high-voltage DC sources by means of linear harvesting and one low-voltage DC source via a boost converter, in the meantime protecting the MEC and providing a regulated output voltage. Energy harvesting from artificial and solar light is then enabled by connecting solar cell 1 to the boost converter input and solar cell 2 to a linear harvesting input, respectively. The LPS is built around the LTC3108 IC by Linear Technology and implements an ultralow-voltage step up converter, with its output connected to the second linear harvesting input of the CPMS, to enable thermal body energy harvesting via an externally connected TEG. The TEG is not integrated onto the antenna to obtain a higher degree of freedom. In this work, a single TEG ( the UltraTec Series UT6,24,F1,5555 from Laird Technologies) is connected to the LPS. Its hot side is tightly attached to the skin of the wearer's arm, whereas its cold side is exposed to the ambient air, in order to harvest thermal body energy based on Seebeck's effect [7]. However, multiple TEGs, potentially stacked, or a heat sink could be used to further increase the amount of harvested energy. To maintain mechanical flexibility of the entire module, the LPS and CPMS are etched on a flexible, ultra-thin polyimide substrate and small electronic components were used and distributed over a larger area than necessary. To maintain antenna performance after hardware integration, it is important to stress that both circuits were oriented in such a way that the feed line remains uncovered and that the two ultra-flexible $200 \mu m$-thick PowerFilm thin film a-Si:Hsolar cells were patterned to fit the antenna's slot. Furthermore, the amount and length of interconnections is minimized by exploiting the antenna cavity as a common DC ground for the energy harvesting hardware and using hollow eyelets to route the positive connection of both solar cells to the power management system. 


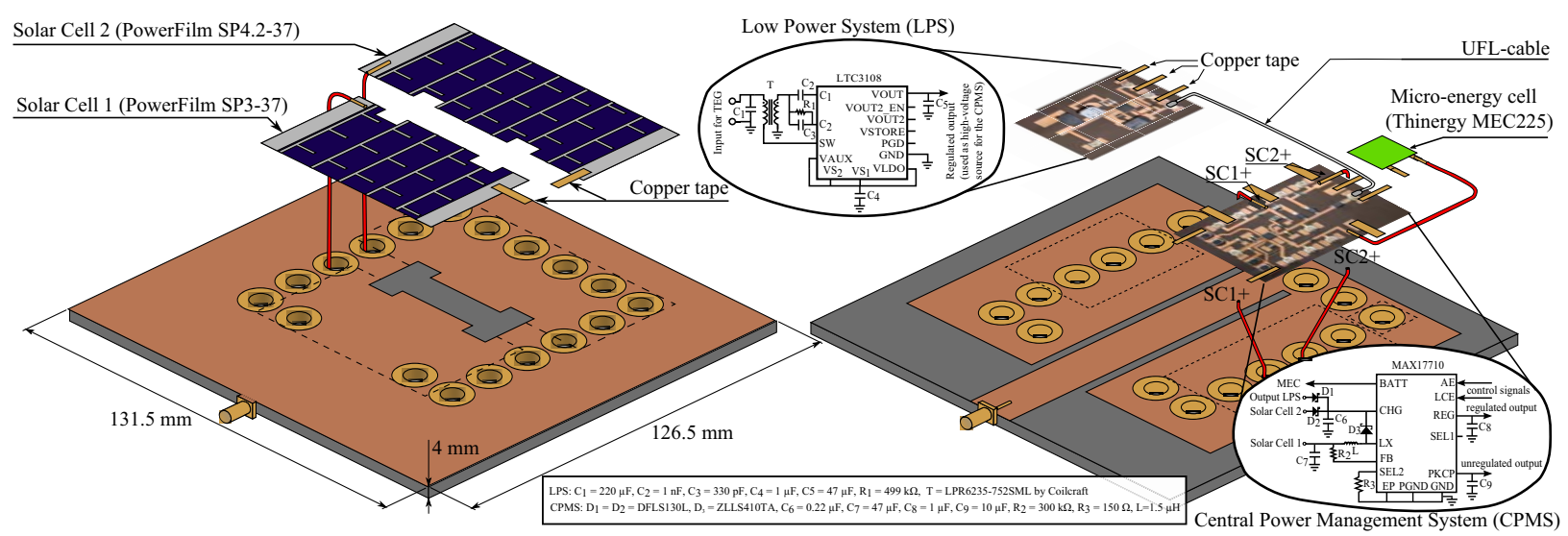

Fig. 2. The wearable SIW cavity-backed textile slot antenna, as described in [9], with two different flexible a-Si:H solar cells glued on top, and the proposed flexible power management module, including a micro-energy cell, integrated at its feed plane.

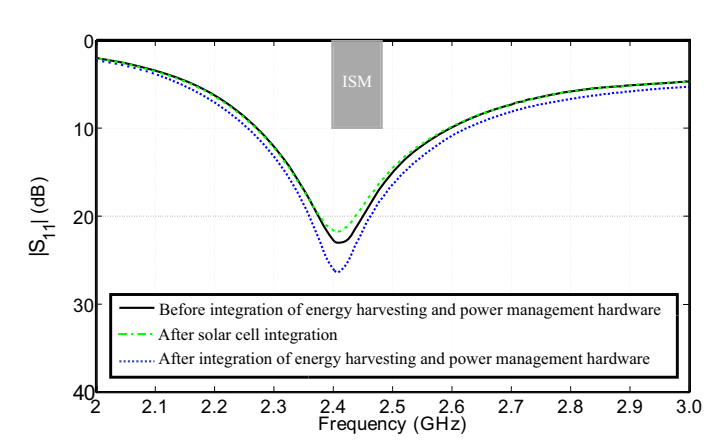

Fig. 3. Measured reflection coefficients of the SIW cavity-backed slot antenna, before and after integration of additional energy harvesting hardware.

\section{Measurement Results}

\section{A. Figures of merit of the textile antenna}

First, the integration procedure is validated by analyzing the influence of integrating energy harvesting and power management hardware on the textile antenna's performance. Therefore, the antenna's reflection coefficient, as a function of frequency, and its radiation pattern, at $2.45 \mathrm{GHz}$, were measured in an anechoic room by means of an orbit/FR 3D positioning system and Agilent's N5242A PNA-X vector network analyzer before the integration of any energy harvesting hardware, after the integration of both solar cells and, finally, when all additional energy harvesting hardware is integrated and all connections are made, as depicted in Fig. 2. The antenna's return loss characteristic from 2.0 to $3.0 \mathrm{GHz}$ is depicted in Fig. 3, whereas Fig. 4 shows the antenna gain in the E- and H-plane at $2.45 \mathrm{GHz}$, for all three setups. These measurements show that the integration of the solar cells, the flexible power management system and MEC only has a minor influence on the antenna's radiation pattern and reflection coefficients. Hence, they prove that the selected antenna is indeed very suitable as an integration platform and enables reducing the amount and length of interconnecting wires by exploiting its properties. The antenna's excellent onbody performance was already demonstrated in [9].

\section{B. Energy supplied by the hybrid energy-harvesting system}

Then, four well-chosen scenarios, each lasting 20 minutes, are considered in a realistic indoor environment to demonstrate the energy harvesting potential of our system in low power

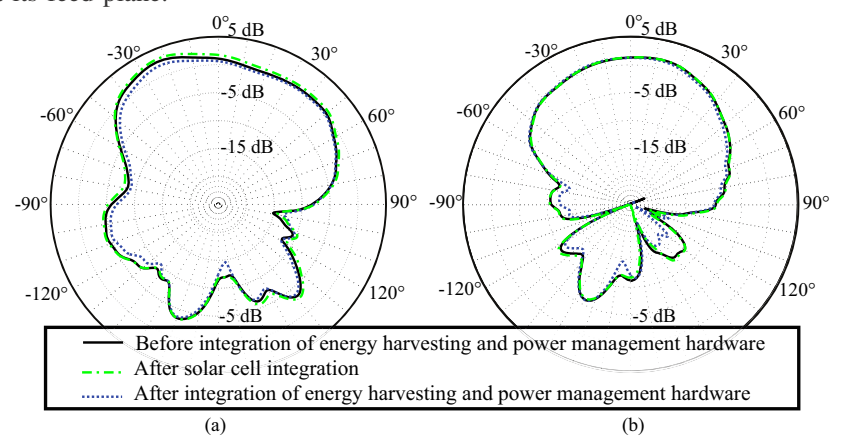

Fig. 4. Measured radiation pattern of the SIW cavity-backed slot antenna at $2450 \mathrm{MHz}$. (a) E-plane; (b) H-plane.

density situations. In each scenario, a test person, with a height of $1 \mathrm{~m} 87 \mathrm{~cm}$, wears the compact, highly-integrated and unobtrusive unit on his chest and the selected TEG on his left arm. During each 20-minutes interval, the power scavenged by each of the three energy harvesters is measured as well as the effectively delivered power to the MEC. Yet, only the power delivered by solar cell 1, the TEG and the sum of both are depicted in Fig. 5 (a) to (d), as it turned out that solar cell 2 in combination with the linear harvesting technique was not able to harvest energy in these indoor scenarios. The first scenario comprises a test person sitting in a $2.4 \mathrm{~m}$ high room with an uniform temperature of $15^{\circ} \mathrm{C}$, no active light sources and no air circulation. Fig. 5 (a) shows that the thermal gradient between room and skin temperature is high enough to harvest energy via the TEG, whereas the absence of light prevents solar cell 1 from scavenging energy. The peaks in scavenged energy by the TEG can be attributed to stretching of the wearer. On average, the TEG was able to generate $108.6 \mu \mathrm{W}$, of which $39.2 \mu \mathrm{W}$ was effectively delivered to the MEC, leading to an overall system efficiency $\eta$ of $36.1 \%$. The second scenario is very similar to the first scenario. However, in the second scenario, fluorescent lamps mounted at the ceiling are turned on and the test person is standing in an upright position. As shown in Fig. 5 (b), the TEG and solar cell 1 are now both able to harvest energy. On average, $106.7 \mu \mathrm{W}$ is recovered by the TEG and $33.2 \mu \mathrm{W}$ by solar cell 1 , resulting in a total harvested power of $139.9 \mu \mathrm{W}$. Eventually, $60.6 \mu \mathrm{W}$ was stored in the MEC, corresponding to $\eta=43.2 \%$. In the third scenario, the room is heated up 

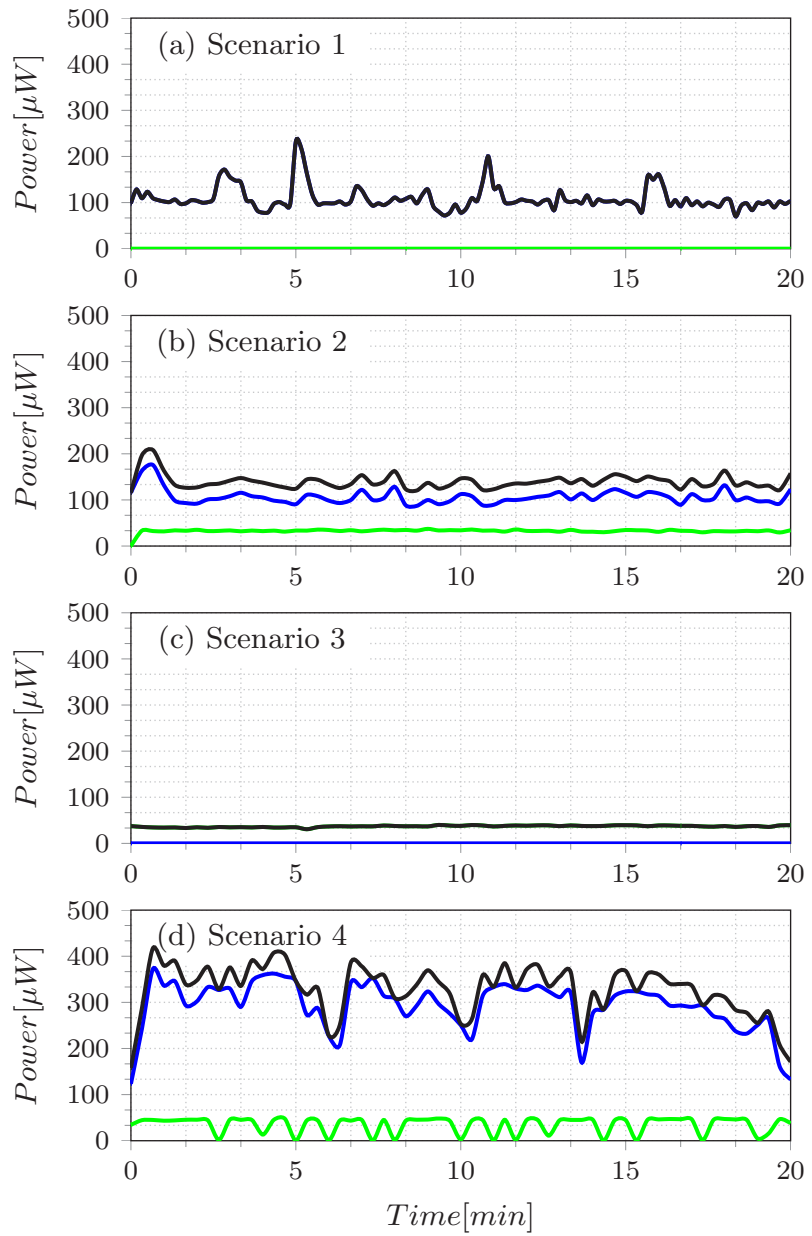

Fig. 5. Power generated by the TEG (blue solid line), solar cell 1 (green solid line) and the sum of both (black solid line) as a function of time for each of the four considered realistic indoor scenarios.

to $25^{\circ} \mathrm{C}$. Fig. 5 (c) indicates that the room temperature is too high for the TEG to recover energy and that only solar cell 1 is capable of harvesting energy. Solar cell 1 produced $37.0 \mu \mathrm{W}$ on average, of which $24.4 \mu \mathrm{W}$ is effectively stored by the MEC. In scenario 4, the test person is walking in a corridor with a height of $2.2 \mathrm{~m}$, a steady temperature of $18^{\circ} \mathrm{C}$, no air circulation and similar fluorescent lights at the ceiling. Fig. 5 (d) clearly demonstrates that, as a result of the dynamic nature of the wearer and the corresponding increase in convection, the power generation by the TEG is substantially increased. Drops in generated power by the TEG and solar cel 1 can be attributed to pauses in which the test person stops walking and the distance between subsequent lamps, respectively. During this 20 -minutes interval, $296.0 \mu \mathrm{W}$ and $34.8 \mu \mathrm{W}$ was generated by the TEG and solar cell 1 on average, respectively. This resulted in a total average power of $152.17 \mu \mathrm{W}$ that could be effectively harvested, leading to $\eta=46.0 \%$. Fig. 5 (a) to (d) clearly evinces the necessity of hybrid energy harvesting in real-life scenarios. Fig. 5 (a) and (c) shows that no power would have been harvested if the user would only have relied on either solar cell 1 or the TEG, respectively. Hence, it reduces the periods during which no energy is harvested. Fig. 5 (b) depicts that hybrid energy harvesting may result in a higher amount of scavenged energy by combining the energy harvested from diverse energy sources. Both effects could lead to either a higher system autonomy or a reduced battery size. An even higher amount of energy could be recovered by solar cell 1 and the TEG in outdoor environments thanks to improved convection and light conditions. In addition, in such an environment, solar cell 2 is also able to scavenge energy. More specific, in case of a turbidity-free sky, placing solar cell 2 perpendicular to the earth surface, pointed in the direction of the sun should enable harvesting an additional $49 \mathrm{~mW}$ [4].

\section{CONCLUSION}

In this paper, the integration of two flexible solar cells, a flexible power management system and a MEC on a wearable SIW cavity-backed textile slot antenna was discussed. Guidelines were provided to select a suitable antenna as integration platform and the pursued integration procedure was outlined, in order to minimize influence on antenna performance, in the meantime guaranteeing the wearer's comfort. Measurements prove that the selected textile antenna is indeed suitable as an integration platform, whereas measurements in four carefullyselected realistic indoor environments demonstrated that a hybrid energy-harvesting approach leads to a higher amount of scavenged energy in a more continuous way.

\section{REFERENCES}

[1] M. A. Hanson, H. C. Powell Jr, A. T. Barth, K. Ringgenberg, B. H Calhoun, J. H. Aylor, and J. Lach, "Body area sensor networks: Challenges and opportunities," Computer, vol. 42, no. 1, pp. 58-65, 2009.

[2] L. Huang, V. Pop, R. de Francisco, R. Vullers, G. Dolmans, H. de Groot, and K. Imamura, "Ultra low power wireless and energy harvesting technologies - an ideal combination," in Proc. IEEE Int. Conf. Commun. Syst., Singapore, 17-19 Nov. 2010, Conference Paper, pp. 295-300.

[3] P. Van Torre, L. Vallozzi, A. Dierck, H. Rogier, and M. Moeneclaey, "Power-efficient body-centric communications," in URSI Benelux Forum, 14 Sept. 2012, Conference Paper, pp. 8-10.

[4] S. Lemey, F. Declercq, and H. Rogier, "Textile antennas as hybrid energy-harvesting platforms," Proc. IEEE, vol. 102, pp. 1833-1857, 2014.

[5] A. Collado and A. Georgiadis, "Conformal Hybrid Solar and Electromagnetic (EM) Energy Harvesting Rectenna," IEEE Trans. Circuits Syst. I, Reg. Papers, vol. 60, no. 8, pp. 2225-2234, Aug 2013.

[6] S. Lemey, F. Declercq, and H. Rogier, "Dual-band substrate integrated waveguide textile antenna with integrated solar harvester," IEEE Antennas Wireless Propag. Lett., vol. 13, no. 1, pp. 269-272, 2014.

[7] M. Lossec, B. Multon, H. Ahmed, and C. Goupil, "Thermoelectric generator placed on the human body: System modeling and energy conversion improvements," Eur. Phys. J, vol. 52, no. 1, pp. 1-10, 2010.

[8] M. Virili, A. Georgiadis, K. Niotaki, A. Collado, F. Alimenti, P. Mezzanotte, L. Roselli, and N. B. Carvalho, "Design and optimization of an antenna with thermo-electric generator (teg) for autonomous wireless nodes," 2014 IEEE RFID Technology and Applications Conference (RFID-TA), pp. 21-25, Sep 8-9 Sept. 2014.

[9] R. Moro, S. Agneessens, H. Rogier, and M. Bozzi, "Wearable textile antenna in substrate integrated waveguide technology," IET Electron. Lett., vol. 48, no. 16, pp. 985-987, 2012.

[10] S. Agneessens, S. Lemey, R. Moro, M. Bozzi, and H. Rogier, "The next generation textile antennas based on substrate integrated waveguide technology," in Proc. of the XXXIth General Assembly and Scientific Symposium of the International Union of Radio Science, Beijing, China, 2014, Conference Paper, pp. 1-4. 